\title{
PROPOSICIÓN DE UN MODELO MATEMÁTICO SIMPLE DE PERSISTENCIA DE HERBICIDAS EN EL SUELO'
}

\author{
RICARDO FUENTES ${ }^{2}$, LUIS UMAÑA ${ }^{3}$, NIMIA MANQUIÁN y RÁUL CRISTT
}

\begin{abstract}
RESUMEN - El estudio propone un modelo simple de persistencia de herbicidas en el suelo basado en una serie de modificaciones al modelo desarrollado por Walker \& Barnes. El modelo que se propone simula la degradación diaria de un herbicida en el suelo, a través del funcionamiento de tres submodelos: a) submodelo que estima la temperatura del suelo, b) submodelo del cálculo del contenido de humedad del suelo y c) submodelo que calcula la degradación del producto. Se entrega una descripción teórica de las modificaciones introducidas al modelo y un detalle del programa computacional en lenguaje BASIC. Se hizo una validación independiente de cada uno de los submodelos modificados y se concluye que todos ellos mejoran su eficiencia de predicción respecto al modelo original. La validación del submodelo de degradación se realizó utilizando información obtenida en campo en dos suelos diferentes de los herbicidas metsulfuron-metil y triasulfuron. Finalmente se concluye que el modelo propuesto sería eficiente en la simulación de la persistencia de estas sulfonilureas en el suelo, utilizando una cinética de primer grado para metsulfuron-metil y una de segundo grado para triasulfuron.
\end{abstract}

Términos para índice: efectos residuales, degradación, humedad, temperatura, simulación.

\section{SIMPLE MATHEMATICAL MODEL PROPOSITION OF HERBICIDE PERSISTENCE IN THE SOIL}

\begin{abstract}
The present study proposes a simple model of persistence of herbicides in soil based on a series of modifications to the model developed by Walker \& Barnes. The model proposed simulates the daily degradation of a herbicide in soil, through three submodels: a) soil temperature submodel, b) soil moisture content submodel and, c) degradation of the product submodel. A theoretical description of the modifications introduced to the model and a detail of the software program in language BASIC are given. An independent validation of each one of the modified submodels was done and it concludes that all of them improve the efficiency of prediction of the original model. The validation of the degradation submodel was made using field obtained information in two different soils from the herbicides metsulfuron-metil and triasulfuron. Finally it is possible to conclude that the proposed model would be efficient in the simulation of the persistence of these sulfonylureas in the soil, using a kinetic first degree model for metsulfuron-mehtyl and one of second degree for triasulfuron.
\end{abstract}

Index terms: residual effects, degradation, humidity, temperature, simulation.

\footnotetext{
${ }^{1}$ Aceptado para publicación en 8 de agosto de 2000 . Trabajo financiado por FONDECYT proyecto 1960928.

${ }^{2}$ Ing. Agr., M.Sc., Cátedra de Malezas y su Control, Instituto Producción y Sanidad Vegetal, Facultad de Ciencias Agrarias, Universidad Austral de Chile (UACH), Casilla 567, Valdivia, Chile. E-mail: rfuentes@uach.cl

${ }^{3}$ Ing. Agr., Instituto Producción y Sanidad Vegetal, Facultad de Ciencias Agrarias, UACH. E-mail: luisuman@telnet.cl

${ }^{4}$ Químico, Instituto Producción y Sanidad Vegetal, Laboratorio Fitoquímica, UACH. E-mail: nmanquia@uach.cl

${ }^{5}$ Químico, Instituto de Medicina Preventiva Veterinaria, UACH. E-mail : rcristi@uach.cl
}

\section{INTRODUCCIÓN}

El uso de modelos de simulación de pesticidas como un instrumento de investigación agronómica han resultado especialmente promisorios para el establecimiento de relaciones entre ciertas variables ecológicas y residualidad de estos productos en el suelo, puesto que permiten una estimación de los procesos a que están sujetos los pesticidas en una forma cuantitativa, en términos de magnitud de su ocurrencia en el tiempo, en función de las condiciones ambientales imperantes. 
En estos últimos años se han desarrollado varios modelos matemáticos para simular la movilidad y persistencia de los herbicidas en el suelo, entre los cuales se puede mencionar los modelos desarrollados por Leistra et al. (1980), Walker \& Barnes (1981) y Shaaban \& Elprince (1989). Entre los más recientes se destacan el LEACH desarrollado por la Universidad de Cornell, USA (Wagenet \& Hutson, 1987; Hutson \& Wagenet, 1992); el modelo PRZM desarrollado por la Agencia de Protección Ambiental de los Estados Unidos de América (Carsel et al., 1984) y su versión modificada PELMO (Klein, 1991); el modelo PESTLA desarrollado en Holanda (Boesten \& Linden, 1991); el modelo CALF (Nicholls et al., 1982) que dio origen a la nueva versión VARLEACH(Walker \& Welch, 1989; Walker \& Hollis, 1994) y la versión más reciente del modelo MACRO descrito por Bergström (1996).

Todos estos modelos permiten realizar predicciones bastante aproximadas de la persistencia de los herbicidas en el suelo, sin embargo, la mayoría de ellos requiere de una gran cantidad de información de entrada altamente específica y sofisticada, no siempre disponible para cualquier zona o región. Además, muchos de estos modelos están basados exclusivamente en una reacción de cinética de primer orden la cual en muchos casos entrega solo una aproximación de la realidad, no siempre extrapolable a cualquier área o condición ambiental.

Teniendo en consideración las características de simplicidad requeridas por los modelos, mencionadas anteriormente, Walker \& Barnes (1981) proponen un modelo sencillo que permite simular la degradación de un herbicida en el suelo utilizando registros diarios de temperatura del aire y pluviometría, constantes de degradación del producto y algunos parámetros geográficos y edáficos del lugar o zona.

El objetivo del estudio fue evaluar y proponer modificaciones al modelo de Walker \& Barnes (1981) validando su eficiencia en la simulación de la persistencia de los herbicidas metsulfuron-metil y triasulfuron en suelos agrícolas del sur de Chile.

\section{MATERIAL Y MÉTODOS}

El estudio tomó como base el modelo propuesto por Walker \& Barnes (1981) en el cual se proponen modificaciones que aumenten la eficiencia de predicción. La estructura base del modelo modificado se describe en el diagrama de flujo de la Fig. 1. En esta se observa que el

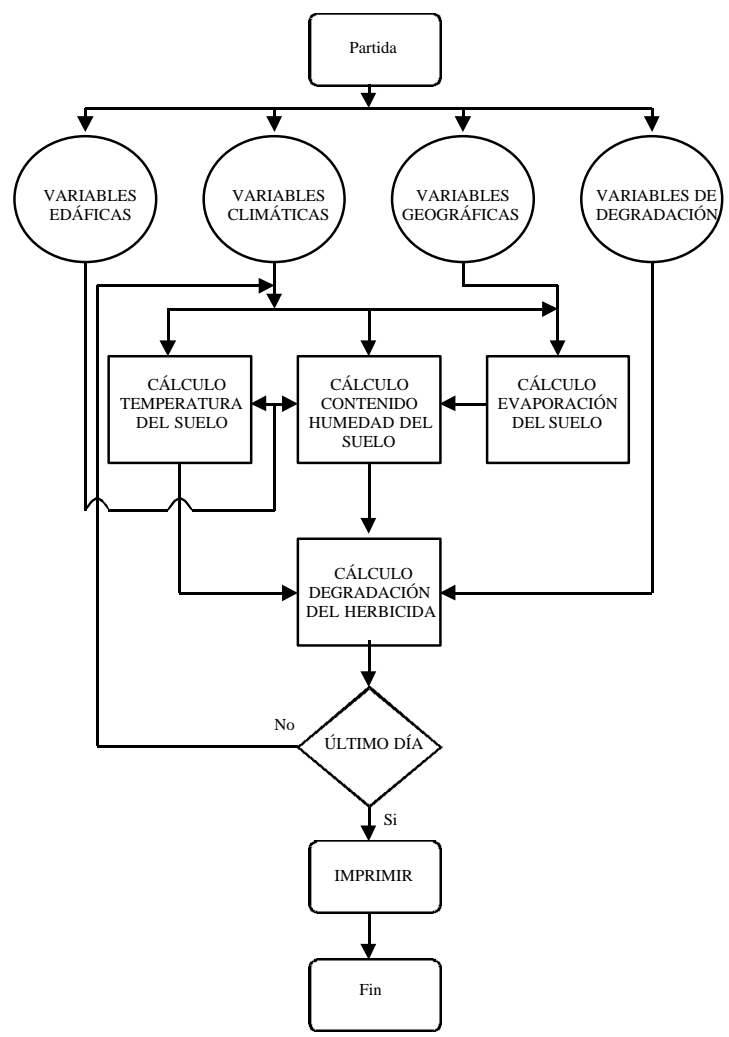

FIG. 1. Diagrama de flujo del modelo propuesto para simular la persistencia de herbicidas en los suelos.

modelo consta de cuatro grupo de variables de entrada, tres submodelos de cálculo y las variables de salidas o resultados. Las modificaciones que se proponen afectan principalmente a algunas ecuaciones que componen cada uno de los submodelos. Las ecuaciones que componen el modelo se presentan en el siguiente programa computacional en lenguaje BASIC:

Descripción del programa BASIC del modelo propuesto para simular la persistencia de herbicidas en el suelo

10 DIM PP(999),MAX(999), MIN(999), MEDIA(999), RANGO (999) , RMED(999), MEDIA2(999), RMED2(999), SMEDIA(999), SMEDIA2(999), SAMP(999), SAMP2(999), SMAX(1999),SMIN(999)

20 DIM EO(365), HUM(365), EVAP(365), PPR(365), $\operatorname{STK}(365), \operatorname{CONC}(365)$ 
$25 C L S$

30 PRINT “ MODELO SIMULACIÓN DE LA PERSISTENCIA DE UN HERBICIDA ENEL SUELO" :PRINT :PRINT

40 INPUT “NOMBRE DEL HERBICIDA :",HERBI\$: INPUT "NOMBRE DEL SUELO A UTILIZAR: "SUELO\$:PRINT :PRINT

100 INPUT; ”ALTITUD (msnm): “, ALT: INPUT “ LATITUD $\left({ }^{\circ}\right):$ ", LAT

110 INPUT; " ${ }^{\circ}$ MES MÁS CÁLIDO (IC): “CAL:INPUT” $T^{o}$ MES MÁS FRIO $\left({ }^{\circ} \mathrm{C}\right)$ : ",FRI

115 DIFTEM $=C A L-F R I: F 2=100-L A T$ : $F 5=0,0023 * A L T$

120 INPUT "NOMBRE ARCHIVO CON DATOS CLIMÁTICOS (Tmax,Tmin,pp):”, DATOS\$

125 PRINT:PRINT "CARACTERÍSTICAS DEL SUELO"

130 INPUT; "PROFUNDIDAD DE PREDICCIÓN (cm): PROF:INPUT " DENSIDAD APARENTE $\left(\mathrm{g} / \mathrm{cm}^{3}\right):$ ", $D A$

140 INPUT ;"CONTENIDO DE HUMEDAD INICIAL (\% P/P): "HUM(O): INPUT “CAPACIDAD DE CAMPO (\% P/P): “,CC

150 PRINT :PRINT"CARACTERÍSTICAS DEL HERBICIDA",

160 INPUT; "CONCENTRACIÓN INICIAL $(\mathrm{mg} / \mathrm{kg})$ : “, CONC(O):INPUT “ ENERGÍA ACTIVACIÓN (cal/mol): ",EA

170 INPUT;" CONSTANTES DE DEGRADACIÓN: $A=$ ",A:INPUT " $B=$ ",B

180 INPUT'TEMPERATURA A QUE SE DETERMINARON LAS CONSTANTES DE DEGRADACIÓN $\left({ }^{\circ} \mathrm{C}\right):{ }^{\prime}, T$

$190 \quad T K=T+273$

500 REM Ingreso de datos climáticos

510 OPEN “I”,\#l,DATOS\$

$520 \quad I=1$

530 WHILE NOT EOF(1)

540 INPUT \#1,MAX(I),MIN(I),PP(I)

$550 \quad I=1: N=N+1$

560 WEND

570 CLOSE \#1

600 REM Cálculo de parámetros temperatura del suelo 604 FOR $I=1$ TO N

$606 \mathrm{~J}=I+1$

608 IF $I=N$ THEN $J=I$

$610 \operatorname{MEDIA}(I)=(M A X(I)+M I N(I)) / 2$

$620 R A N G O(I)=M A X(I)-M I N(l)$

$630 R M E D(I)=R A N G O(I) / 2$

$640 \operatorname{MEDIA2}(I)=(\operatorname{MAX}(J)+\operatorname{MIN}(J)) / 2$
$650 \operatorname{RMED2}(I)=(M A X(J)-M I N(J)) / 2$

$660 \operatorname{SMEDIA}(I)=3,149+(0,676 *(M E D I A(I)))-$ $(0,06 *(R M E D(I)))$

$670 \operatorname{SMEDIA2}(I)=3,149+\left(0,676^{*}(\operatorname{MEDIA2}(I))\right)$ $(0,06 *(R M E D 2(I)))$

$680 \operatorname{SAMP}(I)=2,145-0,1 * M E D I A(I))+$ $(1,162 *(R M E D(I)))$

$690 \operatorname{SAMP} 2(I)=2,145-(0,1 * \operatorname{MEDIA2}(I))+$ $(1,162 *(R M E D 2(I)))$

691 IF MEDIA(I)<12 GOTO 700

$692 \operatorname{SMEDIA}(I)=-11,8+(2,023 *(\operatorname{MEDIA}(I)))+$ $(2,78 *(R M E D(I)))-\left(0,02 *\left(M E D I A(I)^{\wedge} 2\right)\right)$ $\left(0,16^{*}\left(R M E D(I)^{\wedge} 2\right)\right)$

$694 \operatorname{SMEDIA2}(I)=11,8+(2,023 *(M E D I A 2(I)))+$ $\left(2,78^{*}(\operatorname{RMED2}(I))\right)-\left(0,02 *\left(\operatorname{MEDIA2}(I)^{\wedge} 2\right)\right)$ $\left(0,16^{*}\left(R M E D 2(I)^{\wedge} 2\right)\right)$

$696 \operatorname{SAMP}(I)=-13,5+(0,735 * M E D I A(I))+$ $(4,412 *(R M E D(I)))-(0,24 *(R M E D(I) \wedge 2))$

$698 \operatorname{SAMP} 2(I)=-13,5+(0,735 *$ MEDIA2(I) $)+$ $(4,412 *(\operatorname{RMED2}(I)))-\left(0,24 *\left(\operatorname{RMED2}(I)^{\wedge} 2\right)\right)$

700 SMAX(I):SMEDIA(I)+SAMP(I)

$710 \operatorname{SMIN}(I)=\operatorname{SMEDIA2}(I)-\operatorname{SAMP} 2(I)$

$720 \operatorname{SMEDIA}(I)=(\operatorname{SMAX}(I)+\operatorname{SMIN}(I)) / 2$

$730 \operatorname{SAMP}(I)=(\operatorname{SMAX}(I)-\operatorname{SMIN}(I)) / 2$

1000 REM Cálculo de Eo usando ecuaciones de Linacre

$1010 \quad F 1=700 *(M E D I A(I)+(0,006 * A L T))$

$1020 \quad F 2=100-L A T$

$1030 \quad F 5=0,0023 * A L T$

$1040 \quad F 6=0,37 * M E D I A(I)$

$1050 \quad F 7=0,53 * R A N G O(I)$

$1060 \quad F 8=(0,35 *$ DIFTEM $)-10,9$

$1070 \quad F 3=15 *(F S+F 6+F 7+F 8)$

$1080 \quad F 4=80-M E D I A(l)$

$1090 \quad E O(I)=(((F l / F 2)+F 3) / F 4)$

$1100 \quad E O(I)=0,396+(1,121 * E O(I))$

1110 IF $E O(I)=<0$ THEN EO(I) $=0$

$1130 \quad N E X T I$

2000 REM Cálculo del contenido de la humedad del suelo

$2020 C C M=C C^{*} D A^{*} P R O F^{*} 0,1$

$2030 \quad K 4=(0,5 * C C) / 2,176$

$2040 \quad K 3=C C-K 4$

2050 FOR $I=1$ TO N

$2070 S A S=2,303 *(K 3-H U M(I-1)) / K 4$

$2080 \quad S A S=E X P(S A S)$

$2090 \quad A F=0$

2100 IF SAS $<600$ THEN AF $=0,01-0,00002 *(S A S-100)$

2110 IF $S A S<100$ THEN AF $=0,1-0,00129 *(S A S-30)$

2120 IF $S A S<30$ THEN AF $=1-0,031 *(S A S-1)$

2130 IF $S A S<L$ THEN $A F=1$

$2140 \quad \operatorname{EVAP}(I)=E O(I) * A F$ 
5002

5004

5006

$H U M(I-1)=H U M(I-1) * D A * P R O F * 0,1$

IF EVAP(I)>HUM(I-1) THEN EVAP(I)=HUM(I-1)

IF $P P(I)>C C M-H U M(I-1)$ THEN PP(I)=CCM$\operatorname{HUM}(I-1)$

$H U M(I)=H U M(I-l)+P P(I)-E V A P(I)$

$H U M(I)=(H U M(I) * 10) /(D A * P R O F)$

$H U M(I-l)=(H U M(I-l) * 10) /(D A * P R O F)$

NEXT I

REM Cálculo de la temperatura del suelo a profundidad de predicción

FOR $I=1$ TO N

\section{$D A M P=S A M P(I) * E X P(-P R O F / 12,35)$}

$S T E M P=S M E D I A(I)+\left(D A M P * \operatorname{SIN}\left(\left(6,283^{*}(I)\right)-\right.\right.$ (PROF/10,7)))

$\operatorname{STK}(I)=S T E M P+273$

NEXT I

REM Cálculo de la degradación del herbicida

FOR $I=1$ TO N

$V M=A^{*}(H U M(I))^{\wedge} B$

$K l=E A /\left(4,575^{*} T K\right)$

$K 2=K l^{*}(T K-S T K(I)) / S T K(I)$

$V M T=2,303 * K 2$

$V M T=V M * E X P(V M T)$

IF HERBI\$= "TRIASULFURON" THEN HDG= -((CONC(I-1)-2)/VMT*CONC(O))

IF HERBI\$ = "METSULFURON" THEN HDG= $(-(0,6932 * \operatorname{CONC}(I-1) / V M T))$

$C O N C(I)=C O N C(I-l)+H D G$

NEXT 1

$\operatorname{STK}(0)=S T K(l)$

REM Impresión de los resultados

LPRINT "PREDICCIÓN DE LA PERSISTENCIA DEL HERBICIDA ",HERBI\$: LPRINT “APLICADO ENEL SUELO: ",SUELO\$:LPRINT:LPRINT LPRINT ;”ALTITUD (m): “,ALT: LPRINT "LATITUD ( $\left.{ }^{\circ}\right)$ : “,,LAT

LPRINT ; "T MES MÁS CÁLIDO $\left({ }^{\circ} \mathrm{C}\right)$ : “„CAL:LPRINT “T MES MÁS FRIO $\left({ }^{\circ} \mathrm{C}\right)$ : “, FRI LPRINT "NOMBRE ARCHIVO CON DATOS CLIMÁTICOS : “, DATOS\$

5008 LPRINT;" PROFUNDIDADDE PREDICCIÓN (cm): PROF:LPRINT "DENSIDADAPARENTE $\left(\mathrm{g} / \mathrm{cm}^{3}\right):$ ", DA

5010 LPRINT ; "CONTENIDO DE HUMEDAD INICI $A L(\%$ P/P): " $H U M(O):$ LPRINT “ CAPACIDAD DE CAMPO (\% P/P): “,CC

5012 LPRINT :LPRINT”'CARACTERÍSTICAS DEL HERBICIDA"

5014 LPRINT; "CONCENTRACIÓN INICIAL $(\mathrm{mg} / \mathrm{kg})$ : “, CONC(O):LPRINT "ENERGÍA ACTIVACIÓN (cal/mol): “,EA
5016 LPRINT ; "CONSTANTES DE DEGRADACIÓN: ":LPRINT “A: “,,A:LPRINT “B: “,,B

5018 LPRINT "TO DETERMINACIÓN CTES DEGRADACIÓN $\left({ }^{\circ} C\right):$ :" $T$ :LPRINT :LPRINT :LPRINT" DEGRADACIÓN DIARIA DEL HERBICIDA":LPRINT

5020 LPRINT'DIA", "CONCENTRACIÓN", "HUMEDAD", "TEMPERATURA"

5030 LPRINT” “,” HERBICIDA “,”SUELO “,” SUELO”

5040 LPRINT" Conc.Ini.)”," $\left({ }^{\circ} \mathrm{C}\right)$ “:LPRINT

5045 FOR I=0 TO N

5060 LPRINT I,((CONC(I)/CONC(0))*I00),HUM(I), $\operatorname{STK}(I)-273$

5070 NEXTI

6000 END

\section{Submodelo temperatura del suelo}

\section{Modificación propuesta}

El modelo de Walker \& Barnes (1981) propone una estimación de la temperatura del suelo a diferentes profundidades en función de las temperaturas máximas y mínimas del aire, asumiendo que existe una relación lineal de primer grado entre las temperaturas medias y amplitudes térmicas del aire y del suelo. Esta relación lineal fue validada, usando la información de temperaturas del aire y del suelo (2, 5 y $10 \mathrm{~cm}$ de profundidad), durante los años 1990 y 1991, provenientes de las Estaciones Meteorológicas de Punahue e Isla Teja, Valdivia, Chile, ambas de propiedad de la Universidad Austral de Chile. La validación realizada permitió concluir que es aceptable asumir una relación lineal de primer grado entre estas variables, solo cuando las temperaturas medias del aire sean iguales o inferiores a $12^{\circ} \mathrm{C}$ y que considerar una relación lineal de segundo grado cuando las temperaturas del aire superan los $12^{\circ} \mathrm{C}$ aumentaría la predicción del submodelo. Las ecuaciones que mejor describen esta relación $\left(0,921>\mathrm{r}^{2}>0,986\right)$ son las siguientes:

Para temperaturas iguales o inferiores a $12^{\circ} \mathrm{C}$ :

Smed $=3,149+0,676 *$ Med $-0,06 *$ Amp

Samp $=2,149-0,1 * \mathrm{Med}+1,162 *$ Amp

Para temperaturas superiores a $12^{\circ} \mathrm{C}$ :

Smed $=1+2,023 * \mathrm{Med}+2,78 * \mathrm{Amp}-0,02 * \mathrm{Med}^{2}-$ $0,16^{*} A m p^{2}$ 
Smed $=-13,5+0,735 * \mathrm{Med}+4,412 * \mathrm{Amp}-$

$0,24 * A m p^{2}$

Donde: Smed $=$ temperatura media diaria del suelo $\left({ }^{\circ} \mathrm{C}\right)$; Samp $=$ amplitud diaria de la temperatura en la superficie del suelo $\left({ }^{\circ} \mathrm{C}\right) ; \mathrm{Med}=$ temperatura media diaria del aire $\left({ }^{\circ} \mathrm{C}\right)$; y $A m p=$ amplitud térmica diaria del aire $\left({ }^{\circ} \mathrm{C}\right)$.

Información utilizada en la validación

La evaluación de las ecuaciones de regresión térmicas tanto del submodelo propuesto por Walker \& Barnes (1981) como para las modificaciones propuestas al modelo, se validaron comparando los valores estimados por ambos modelos comparados con las temperaturas máximas y mínimas diarias del aire y las temperaturas medias del suelo a $5 \mathrm{~cm}$ de profundidad, registradas por la Estación Meteorológica Isla Teja del Instituto de Geociencias de la Universidad Austral, Valdivia, Chile, durante el año 1996.

\section{Submodelo contenido de humedad del suelo}

Modificación propuesta

El modelo original de Walker \& Barnes (1981) asume que el suelo tiene un perfil uniforme y que la acumulación de humedad no depende del subsuelo. El agua que entra al sistema proveniente de las lluvias, se suma al contenido de agua inicial del suelo hasta el límite de capacidad de campo; niveles superiores a este contenido de humedad son ignorados. Las pérdidas de agua del suelo son calculadas por multiplicación de la evaporación de agua desde una superficie abierta por un factor que depende de la tensión por la cual es retenida el agua en el suelo. Las pérdidas diarias de humedad por evaporación del suelo se estiman a través de una serie de ecuaciones derivadas por Linacre (1977), las que permiten calcular la evaporación a través de las temperaturas máximas y mínimas diarias del aire, altitud y latitud del sitio. La validación de estas ecuaciones de Linacre fue realizada en Wellesbourne, Inglaterra por los autores Walker \& Barnes (1981), las que al compararlas en un análisis de regresión lineal con los valores medidos, se observó que éstos en general eran más bajos que los estimados $\left(r^{2}=0,805\right)$.

En este estudio se propone la ecuación (5) que permitió una mejor estimación de la evaporación $\left(\mathrm{r}^{2}=0,982\right)$ y que fue validada en las zonas agroecológicas de La Unión y Riñihue, ambas ubicadas en la Décima Región de Chile durante la temporada 1997.
$E m=1,48+0,25 * E c$

Donde: $E m$ = evaporación medida en un evaporímetro de bandeja clase A (mm/día) y $E c$ = evaporación calculada usando las ecuaciones de Linacre (1977).

Por otra parte, se incluyó otra restricción al modelo de Walker \& Barnes (1981), la cual consideró que las pérdidas de humedad (evaporación) no pueden ser superiores al contenido total de humedad existente en el suelo. La estimación del contenido de humedad en el suelo estaría representada por la ecuación (6).

Hum $=$ Huma + PP - Evap

Donde: Hum = contenido de humedad del suelo diario $(\mathrm{mm}) ;$ Huma $=$ contenido de humedad del suelo el día inmediatamente anterior $(\mathrm{mm}) ; P P=$ precipitación diaria $(\mathrm{mm}) ;$ y Evap = evaporación diaria del suelo $(\mathrm{mm})$.

\section{Información utilizada en la validación}

Los datos de campo empleados para la validación de los submodelos de contenido de humedad en el suelo, tanto para el modelo de Walker \& Barnes (1981) como para el modificado, corresponden a los valores observados por Olea (1996), en un suelo Palehumults typic serie Cudico durante la temporada agrícola 1995 y en un suelo Dystrandeps typic serie Malihue durante la temporada agrícola 1994/1995. La información meteorológica correspondiente fue obtenida en la Estación Climatológica de propiedad de la empresa COLUN, ubicada en La Unión a una distancia de $3 \mathrm{~km}$ en línea recta de las observaciones obtenidas para el suelo Cudico; y en la Estación Metereológica de Punahue, de propiedad de la Universidad Austral de Chile, ubicada a $500 \mathrm{~m}$ del lugar de ubicación del suelo Malihue.

\section{Submodelo de degradación del herbicida}

\section{Modificación propuesta}

La degradación de un pesticida en el suelo es proporcional a la concentración del producto y puede ser interpretado usando la ecuación (7), según Walker \& Barnes (1981), usando una reacción de primer orden.

$C=C o * e^{-K^{*} t}$

Donde: $\mathrm{C}=$ concentración del pesticida en el tiempo $\mathrm{t}$; $\mathrm{Co}=$ concentración inicial; y $\mathrm{K}=$ constante de degradación.

Pesq. agropec. bras., Brasília, v.35, n.12, p.2317-2328, dez. 2000 
A partir de la ecuación (7) se puede calcular el tiempo en que la concentración es igual al $50 \%$ de la concentración inicial o vida media y la tasa de degradación diaria del producto (Walker \& Barnes, 1981).

En este estudio se propone incluir en el modelo original de Walker \& Barnes (1981) un padrón cinético de segundo grado, ya que el de primer orden no siempre es eficiente en la estimación de la persistencia de algunos pesticidas. En ese sentido, Hamaker (1972) ha sugerido una curva empírica de degradación que permite estimar el orden de la reacción que tendría la ecuación (8).

$$
C=\left(C o^{1-n}+(n-1) * K * t\right)^{\frac{1}{1-n}}
$$

Donde: $\mathrm{C}=$ concentración residual en el tiempo $\mathrm{t}$; Co = concentración inicial; $\mathrm{K}=$ constante de degradación; y $\mathrm{n}=$ orden aparente de la reacción.

De la ecuación (8), con un valor $n=2$, se deriva la ecuación de segundo orden (9) para la degradación de pesticidas.

$\frac{d C}{d t}=-K^{*} C^{2}$

A partir de la ecuación (8), se determina la vida media del producto (VM) en una reacción de segundo orden, siendo esta la ecuación (10) la que representaría este valor.

$$
V M=\frac{1}{K * C o}
$$

De esta manera se obtiene la ecuación (11), que representa el incremento en la degradación de pesticidas con una reacción de segundo orden.

$\frac{d C}{d t}=-\frac{C^{2}}{V M * C o}$

Información utilizada en la validación

Los datos que se emplearon para validar el modelo propuesto, corresponden a los valores de campo obtenidos por Thurn-Valsassina (1998) para residuos totales en los suelos serie Cudico y serie Malihue, para los herbicidas metsulfuron-metil y triasulfurón. La serie Cudico, corresponde a un suelo rojo arcilloso, gran grupo Palehumults typic, ubicado en $\operatorname{los} 40^{\circ}$ L.S. y $73^{\circ} 6^{\prime}$ L.O.; y la serie Malihue, corresponde a un suelo derivado de cenizas volcánicas, gran grupo Dystrandeps typic, ubicado en los 3951' L.S. y 72³0' L.O. (Tosso, 1985). Los experimentos, en ambos suelos, se realizaron entre mayo a diciembre de 1997 y el muestreo se efectuó a los 0, 14, 28, 62, 124 y 220 días después de la aplicación en ambos herbicidas, a una profundidad de $10 \mathrm{~cm}$. La determinación de residuos totales se hizo mediante cromatografía de gas-líquido con detector nitrógeno-fósforo (NPD) de acuerdo a lo descrito por Fuentes et al. (1998).

Los datos climatológicos de lluvia, temperaturas máximas y mínimas diarias que se utilizaron en la validación del submodelo de degradación, correspondieron a observaciones hechas, en igual período del año 1997, por la planta COLUN, en La Unión, ubicada a $3 \mathrm{~km}$ del experimento en el suelo Cudico; y por la Estación Limnológica de Riñihue, ubicada a $10 \mathrm{~km}$ de 1 los experimentos establecidos en el suelo Malihue. Para el cálculo de la energía de activación de los herbicidas y las constantes A y B de Walker (1987), se estimó la vida media de los herbicidas con los datos de degradación en los suelos Cudico y Malihue obtenidos en cámara de incubación a 10, 20 y $30^{\circ} \mathrm{C}$ con 20,60 y $90 \%$ de humedad, evaluando los residuos por cromatografía gas-líquido NPD a los 0, 15, 30, 60, 120 y 210 días después de la aplicación (Fuentes et al., 1998). En el Cuadro 1 se indican los parámetros iniciales requeridos por el modelo para los dos herbicidas y ambos suelos empleados en la validación.

\section{Evaluación de la bondad de ajuste de los submodelos}

La eficiencia de predicción entre los valores estimados por los diferentes submodelos y los valores observados, fue determinada a través de una análisis de regresión lineal; un test de concordancia de Pearson o prueba de $\chi^{2}$ (Steel \& Torrie, 1995); y el coeficiente de forma (CF) e índice de eficiencia de modelación (IEM) descrito por Walker et al. (1996).

\section{RESULTADOS Y DISCUSIÓN}

\section{Validación del submodelo temperatura del suelo}

En la Fig. 2 se muestran los valores de temperatura medias del suelo a $5 \mathrm{~cm}$ de profundidad del suelo observados y simulados de acuerdo al modelo de 
CUADRO 1. Parámetros iniciales utilizados para ser usados en el modelo de Walker \& Barnes (1981) y en el modelo propuesto.

\begin{tabular}{|c|c|c|c|c|}
\hline \multirow[t]{3}{*}{ Variables requeridas } & \multicolumn{4}{|c|}{ Valor del parámetro } \\
\hline & \multicolumn{2}{|c|}{ Metsufuron-metil } & \multicolumn{2}{|c|}{ Triasulfuron } \\
\hline & Cudico & Malihue & Cudico & Malihue \\
\hline \multicolumn{5}{|l|}{ Variables geográficas } \\
\hline Altitud (m) & 35 & 230 & 35 & 230 \\
\hline Latitud $\left({ }^{\circ}\right)$ & 40,25 & 39,8 & 40,25 & 39,8 \\
\hline $\mathrm{T}^{\mathrm{o}}$ media mes más cálido $\left({ }^{\circ} \mathrm{C}\right)$ & 19,2 & 15,7 & 19,2 & 15,7 \\
\hline $\mathrm{T}^{\mathrm{o}}$ media mes más frío $\left({ }^{\circ} \mathrm{C}\right)$ & 8,1 & 5,4 & 8,1 & 5,4 \\
\hline \multicolumn{5}{|l|}{ Variables edáficas } \\
\hline Profundidad predicción (cm) & 10 & 10 & 10 & 10 \\
\hline Densidad aparente $(\mathrm{g} / \mathrm{cc})$ & 1,01 & 0,86 & 1,01 & 0,86 \\
\hline Capacidad de campo (\%) & 47 & 104 & 47 & 104 \\
\hline Humedad inicial suelo (\%) & 42 & 72 & 42 & 72 \\
\hline \multicolumn{5}{|l|}{ Variables de degradación } \\
\hline $\begin{array}{l}\text { Concentración inicial de } \\
\text { herbicida ( } \mathrm{mg} / \mathrm{kg} \text { suelo) }\end{array}$ & 4 & 4 & 4 & 4 \\
\hline Energía activación (cal/mol) & 5455 & 4216 & 12740 & 4003 \\
\hline Constante A de Walker & 275 & 168 & 622 & 438 \\
\hline Constante B de Walker & $-0,36$ & $-0,22$ & $-0,80$ & $-0,51$ \\
\hline
\end{tabular}

Walker \& Barnes (1981) y según el modelo propuesto.

Estos resultados muestran que ambos modelos representan en buena forma la tendencia térmica promedio real a través del año, sin embargo, los dos modelos simulan valores diarios con mayores fluctuaciones que las variaciones térmicas reales dentro de cada período. El modelo de Walker \& Barnes, en general, tiende a producir mayores desviaciones entre los valores observados y simulados que el modelo propuesto, llegando a producir desvíos de hasta $6^{\circ} \mathrm{C}$ a $8^{\circ} \mathrm{C}$. Además, este modelo tiende a sobrestimar los valores de temperatura diarias del suelo superiores a los $15^{\circ} \mathrm{C}$, que según la Fig. 2, correspondería al período comprendido entre diciembre a marzo. El modelo propuesto, en cambio, tiende a subestimar los valores de temperaturas en el suelo durante estos meses de verano, sin embargo, el modelo en general presenta desviaciones no superiores a $\operatorname{los} 5^{\circ} \mathrm{C}$, respecto a los valores.

Los resultados del análisis estadísticos para evaluar la bondad de ajuste de ambos submodelos se presentan en el Cuadro 2. La regresión lineal efectuada entre los valores observados y simulados con ambos submodelos fue significativa $(\mathrm{p}<0,005)$, indicando que ambas herramientas permiten predecir aceptablemente la temperatura del suelo a $5 \mathrm{~cm}$ de profundidad, a través de los valores de temperaturas
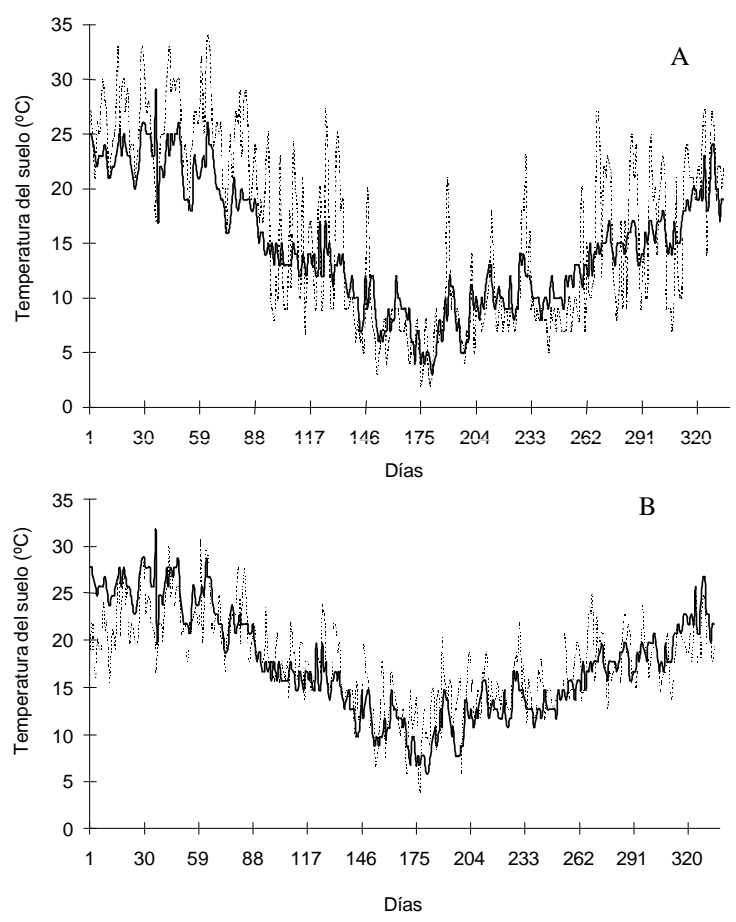

FIG. 2 Temperaturas medias diarias a $5 \mathrm{~cm} \mathrm{de}$ profundidad del suelo observadas $(-)$ y simuladas (.....) por el modelo de Walker \& Barnes (1981) (A) y por el modelo propuesto (B). Estación Meteorológica Isla Teja, Valdivia $(1=1$ de enero 1996). 
máximas y mínimas diarias del aire. Sin embargo, el submodelo propuesto presentó un coeficiente de determinación $\left(\mathrm{r}^{2}=0,756\right)$ ligeramente superior al obtenido para el modelo de Walker \& Barnes (1981) $\left(r^{2}=0,684\right)$, un valor para la pendiente (b) mas cercano a 1 y un valor para el intercepto (a) más próximo a cero. Por otra parte, el test de concordancia $\left(\chi^{2}\right)$ demostró que el modelo propuesto no presentó diferencias significativas entre los valores observados y simulados. Estos resultados son confirmados mediante los coeficientes de bondad de ajuste indicados en el Cuadro 2, que corroboran que el modelo propuesto presenta, respecto al modelo de Walker \& Barnes (1981), una mayor similitud en la forma de la distribución de la curva predecida y observada, a través de un coeficiente de forma (CF) más cercano uno; y una menor desviación entre los datos reales y simulados, reflejados en un índice de eficiencia de modelación (IEM) más próximo al valor 1 .

Lo anteriormente expuesto permite afirmar que las modificaciones incorporadas al submodelo de temperatura del suelo de Walker \& Barnes (1981) aumentarían su eficiencia de predicción. Esta mayor eficiencia sería consecuencia de las variaciones introducidas en las regresiones térmicas (Ecuaciones 1, 2, 3 y 4), en las que se establece que al usar las temperaturas máximas y mínimas del aire como base para la estimación de la temperaturas del suelo, la relación entre estas variables, no sería siempre lineal como lo establece Walker \& Barnes (1981), sino que sería lineal con temperaturas del aire inferiores a $12^{\circ} \mathrm{C}$ y de segundo grado cuando estas son iguales o superiores a $12^{\circ} \mathrm{C}$.

\section{Validación del submodelo de contenido de humedad del suelo}

Los contenidos de humedad en el suelo simulados por ambos modelos y los observados en los suelos Cudico 1995 y Malihue 1994/95, se presentan en la Fig. 3. Estos resultados muestran que los dos modelos evaluados reflejan eficientemente la tendencia seguida por los valores reales de contenido de humedad observados a los $5 \mathrm{~cm}$ de profundidad del suelo a través del tiempo, no determinándose gráficamente marcadas diferencias entre ellos, en ambos suelos. En condiciones de suelos con mayor capacidad de retención de agua como Malihue 1994/95 es posible observar que ambos modelos tienden a sobrestimar los valores de predicción y en el suelo de menor capacidad de retención de humedad como Cudico 1995, los dos modelos tienden a subestimar los contenidos de humedad en el suelo, especialmente en los períodos de menor precipitación como son los meses de enero y febrero.

La evaluación de la eficiencia de predicción de los modelos a través de los diferentes parámetros estadísticos se presenta en el Cuadro 3. El análisis de regresión y los coeficientes de bondad de ajuste (CF y IEM) entre los valores observados y simulados, muestran que los dos modelos permiten una buena predicción del contenido de humedad a $5 \mathrm{~cm}$ de profundidad, no existiendo marcadas diferencias entre ellos. Sin embargo, el test de concordancia $\left(\chi^{2}\right)$ demostró claramente que los valores estimados por el modelo propuesto no difieren significativamente de los valores reales medidos del contenido de humedad en el suelo en el conjunto de observaciones analizadas, no así el modelo de Walker \& Barnes (1981) donde las diferencias entre los valores estimados y observados fueron significativas $\left(\chi^{2}>14,23\right)$.

La incorporación de un balance hídrico como el descrito en la Ecuación (6) y la nueva estimación de la regresión incorporada en el modelo propuesto en este estudio, Ecuación (5), para corregir la

CUADRO 2. Resultados del test de concordancia, análisis de regresión lineal y coeficientes de bondad de ajuste entre los valores observados y estimados para temperatura media en el suelo $(5 \mathrm{~cm}$ profundidad) por el submodelo de Walker \& Barnes (1981) y por el modelo propuesto.

\begin{tabular}{lcc}
\hline Parámetro & \multicolumn{2}{c}{ Modelo } \\
\cline { 2 - 3 } & $\begin{array}{c}\text { Walker \& } \\
\text { Barnes }\end{array}$ & Propuesto \\
\hline Test de concordancia & & \\
$\quad$ Valor de $\chi^{2}$ & 408 & 243 \\
Coeficientes de regresión lineal & & \\
$\quad$ Coeficiente determinación ( $\left.{ }^{2}\right)$ & 0,684 & 0,756 \\
$\quad$ Pendiente (b) & 0,697 & 1,267 \\
$\quad$ Intercepto (a) & 4,752 & $-2,600$ \\
Coeficientes bondad de ajuste & & \\
$\quad$ Coeficiente de forma (CF) & 0,47 & 1,40 \\
Indice eficiencia modelaje (IEM) & 0,35 & 0,60 \\
\hline
\end{tabular}

${ }^{1}$ Valor $\chi^{2}$ a $95 \%$ de concordancia $=291,7$ con $n=333$. 
Cudico
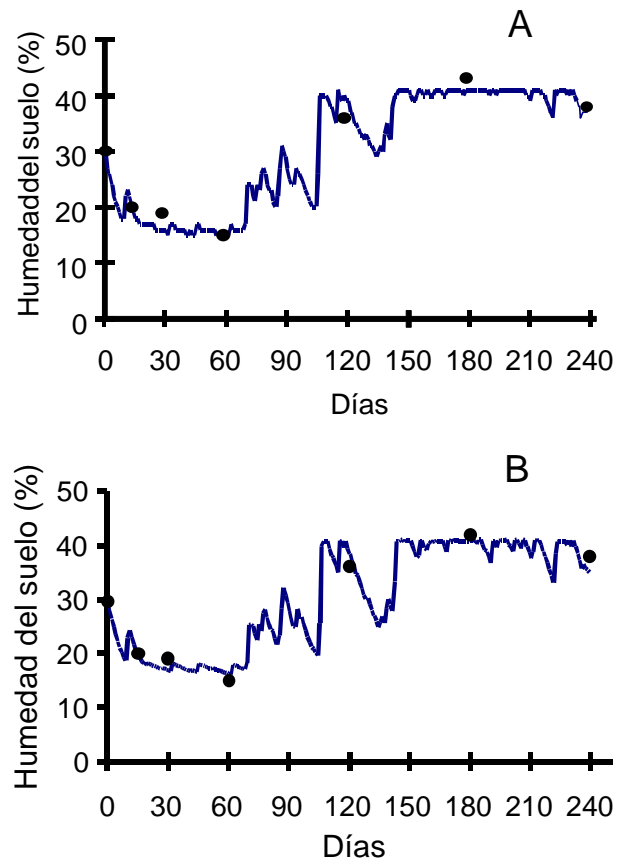

Malihue

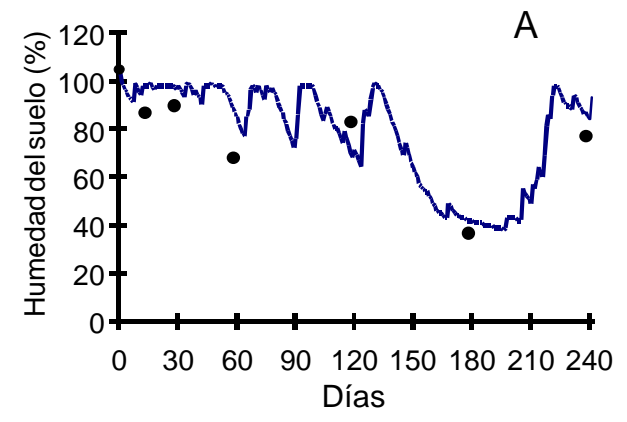

B

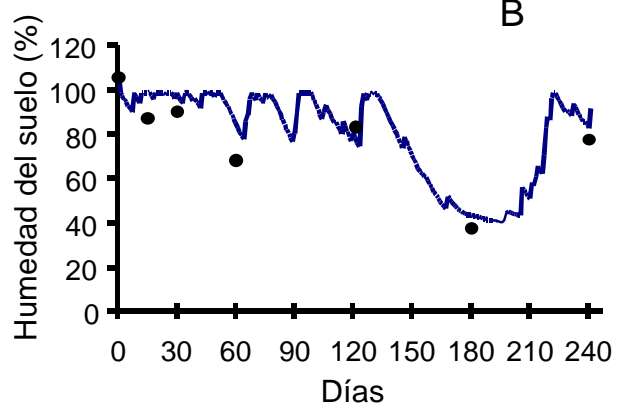

FIG. 3. Contenido de humedad del suelo observados (•) y simulados (_) por el modelo de Walker \& Barnes (1981) (A) y por el modelo propuesto (B), a $5 \mathrm{~cm}$ de profundidad en los suelos Cudico $1995(1=6$ enero 1995) y Malihue 1994/95 $(1=3$ septiembre 1994).

CUADRO 3. Resultados del test de concordancia, análisis de regresión lineal y coeficientes de bondad de ajuste entre los valores observados y estimados para contenido de humedad diario en el suelo $(5 \mathrm{~cm}$ profundidad) por el submodelo de Walker \& Barnes (1981) y por el modelo propuesto, en los suelos Cudico 1995 \& Malihue 1994/95.

\begin{tabular}{lcc}
\hline Parámetro & \multicolumn{2}{c}{ Modelo } \\
\cline { 2 - 3 } & $\begin{array}{c}\text { Walker \& } \\
\text { Barnes }\end{array}$ & Propuesto \\
\hline Test de concordancia & & \\
$\quad$ Valor de $\chi^{2}$ & 18,5 & 9,6 \\
$\quad$ Coeficientes de regresión lineal & & \\
$\quad$ Coeficiente determinación ( $\left.{ }^{2}\right)$ & 0,978 & 0,988 \\
$\quad$ Pendiente (b) & 1,131 & 1,114 \\
$\quad$ Intercepto (a) & $-3,70$ & $-4,10$ \\
Coeficientes bondad de ajuste & & \\
$\quad$ Coeficiente de forma (CF) & 0,76 & 0,79 \\
$\quad$ Indice eficiencia modelaje (IEM) & 0,93 & 0,97 \\
\hline
\end{tabular}

\footnotetext{
${ }^{1}$ Valor $\chi^{2}$ a 95\% de concordancia $=14,2$ con $n=26$.
}

subestimación de la evaporación de humedad desde el suelo calculada mediante las ecuaciones de Linacre (1977) en el modelo de Walker \& Barnes (1981), aumentarían la eficiencia de predicción del contenido de humedad del suelo, considerando cualquier época del año.

\section{Validación del submodelo de degradación}

La simulación de la degradación de los herbicidas en el suelo se realizó utilizando solo los submodelos de temperatura y contenido de humedad en el suelo propuesto, validados anteriormente e incluyendo cinéticas de primer y segundo orden.

En la Fig. 4 se presentan los valores observados y simulados para la persistencia de metsulfuron-metil en los suelos Cudico 1997 y Malihue 1997, considerando un padrón de degradación de primer y segundo grado. En general se observa gráficamente que tanto la cinética de primer como de segundo orden 
en el modelo propuesto tienden a subestimar la concentración de residuos de metsulfuron-metil, en ambos suelos, durante los primeros 30 días después de la aplicación (DDA), siendo ligeramente inferior la subestimación al emplear un modelo de primer grado. A los 220 DDA, en cambio, el empleo de una cinética de degradación de primer orden predice casi con exactitud la concentración determinada en el campo para este herbicida en los dos suelos, observándose una clara subestimación de los valores simulados cuando se empleó una función de segundo orden.

Los valores observados y simulados de la concentración de triasulfuron en los suelos Cudico y Malihue durante la temporada 1997, se presentan
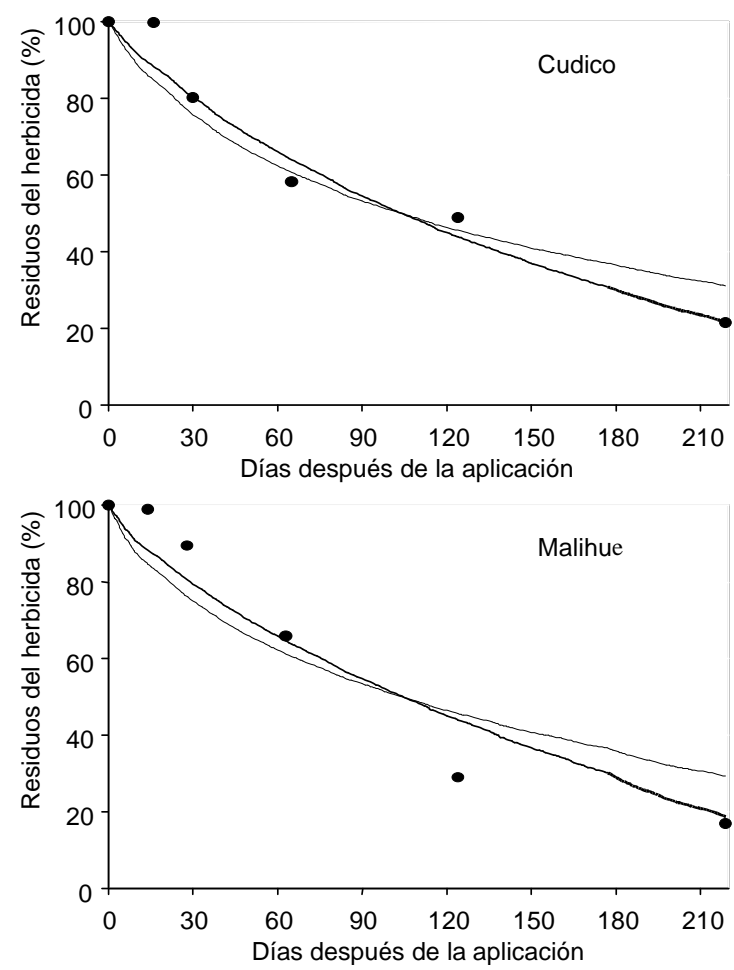

FIG. 4. Residuos totales (\% dosis inicialmente aplicada) de metsulfuron-metil observados (•) y simulados por el modelo propuesto en una cinética de primer orden $(-)$ y de según orden ( $)$ en el suelo Cudico $(1=7$ mayo 1997 ) y Malihue 1997 (1 = 5 de mayo 1997). en la Fig. 5. Estos resultados muestran gráficamente algunas diferencias entre los suelos en la magnitud de los valores estimados por el modelo respecto a los valores reales, especialmente en los primeros días post-aplicación del producto. En el suelo Cudico, ambas cinéticas de degradación, subestiman la concentración simulada del herbicida en los primeros 30 DDA y en el suelo Malihue, en cambio, existió una subestimación de los valores predecidos durante este período. Sin embargo, a los 120 y 220 DDA, la cinética de segundo orden permite una mayor eficiencia al modelo en la predicción de los residuos de triasulfuron, tanto en el suelo Cudico como en Malihue. El modelo de degradación de primer grado

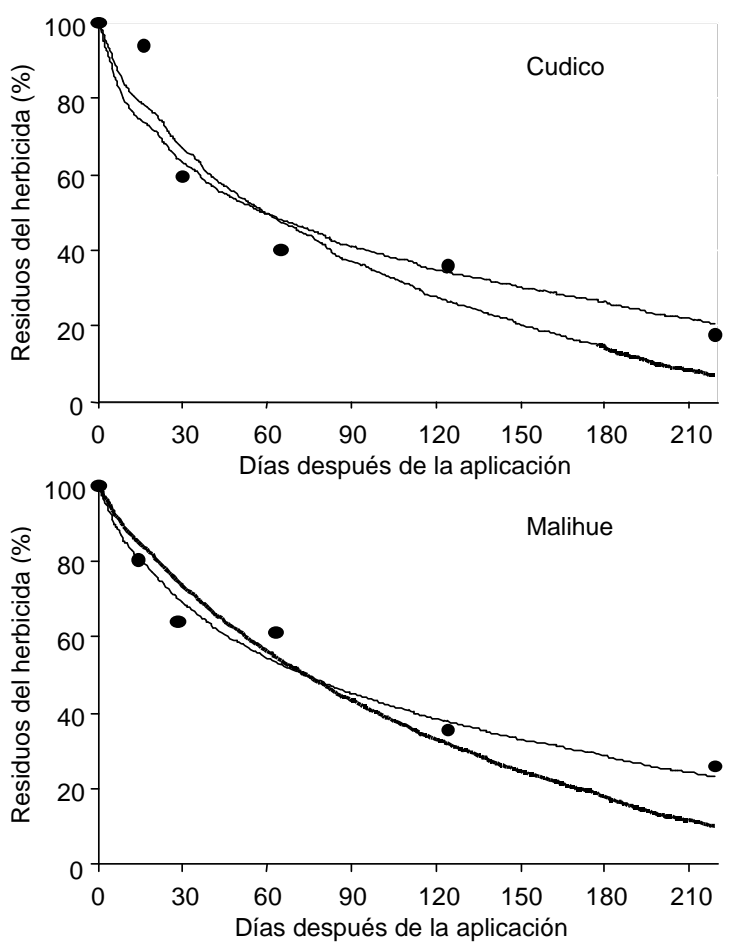

FIG. 5. Residuos totales (\% dosis inicialmente aplicada) de triasulfuron observados (6) y simulados por el modelo propuesto en una cinética de primer orden $(-)$ y de según orden ( ) en el suelo Cudico $(1=7$ mayo 1997$)$ y Malihue 1997 ( $1=5$ de mayo 1997). 
tiende a predecir, durante este último período, concentraciones de residuos bastante mas bajas que las observadas en condiciones de campo.

Los parámetros estadísticos determinados para evaluar la eficiencia de predicción del modelo propuesto se indican en el Cuadro 4. El test de concordancia demostró que no existieron diferencias significativas entre los residuos estimados y los observados, tanto para metsulfuron-metil como para triasulfuron, en los dos suelos estudiados, utilizando cualesquiera de las dos cinéticas de degradación analizadas. Sin embargo, tanto el análisis de regresión como los coeficientes de bondad de ajuste, muestran una mayor eficiencia de simulación del modelo de primer orden para metsulfuron-metil y de segundo orden para triasulfuron, ya que presentaron un valor de pendiente (b) más cercano a 1, un intercepto (a) casi igual a cero y un valor tanto para el coeficiente de forma (CF) como para el índice de eficiencia de modelación (IEM) más cercano a 1. El padrón de degradación de primer orden ya sido reportado mara metsulfuron-metil por Blair \& Martin (1988) y James et al. (1995).

CUADRO 4. Resultados del test de concordancia, análisis de regresión lineal y coeficientes de bondad de ajuste entre los valores observados y estimados de persistencia de residuos de metsulfuron-metil y triasulfuron en el suelo $(10 \mathrm{~cm}$ profundidad) por el modelo propuesto con una cinética de primer y segundo orden en los suelos Cudico 1997 y Malihue 1997.

\begin{tabular}{|c|c|c|c|c|}
\hline \multirow{2}{*}{ Parámetro } & \multicolumn{2}{|c|}{ Metsulfuron-metil } & \multicolumn{2}{|c|}{ Triasulfuron } \\
\hline & $\begin{array}{c}1^{\text {er. }} \\
\text { orden }\end{array}$ & $\begin{array}{c}2^{\text {do. }} \\
\text { orden }\end{array}$ & $\begin{array}{l}1^{\text {er. }} \\
\text { orden }\end{array}$ & $\begin{array}{c}2^{\text {do. }} \\
\text { orden }\end{array}$ \\
\hline \multicolumn{5}{|l|}{$\overline{\text { Test de concordancia }}{ }^{1}$} \\
\hline Valor de $\chi^{2}$ & 0,25 & 0,34 & 0,42 & 0,32 \\
\hline \multicolumn{5}{|l|}{ Coeficientes de regresión lineal } \\
\hline Coeficiente determinación $\left(\mathrm{r}^{2}\right)$ & 0,902 & 0,900 & 0,900 & 0,932 \\
\hline Pendiente (b) & 0,89 & 0,76 & 0,83 & 0,96 \\
\hline Intercepto (a) & 0,09 & 0,17 & 0,15 & 0,08 \\
\hline \multicolumn{5}{|l|}{ Coeficientes bondad de ajuste } \\
\hline Coeficiente de forma (CF) & 1,13 & 1,51 & 0,97 & 1,32 \\
\hline Índice eficiencia modelaje (IEM) & 0,89 & 0,87 & 0,85 & 0,87 \\
\hline
\end{tabular}

${ }^{1}$ Valor $\chi^{2}$ a 95\% de concordancia $=8,67$ con $\mathrm{n}=17$.
Los resultados expuestos permiten confirmar que el modelo propuesto sería eficiente en la simulación de la persistencia de las sulfonilureas en el suelo, utilizando una cinética de primer orden para el herbicida metsulfuron-metil y una de segundo orden para triasulfuron.

\section{CONCLUSIONES}

1. Las modificaciones propuestas a los submodelos desarrollados por Walker \& Barnes (1981) para simular la persistencia de los herbicidas en el suelo permiten aumentar la su eficiencia de predicción del contenido de humedad del suelo y de las temperaturas del suelo usando como información base las lluvias y las temperaturas máximas y mínimas diarias del aire.

2. El modelo propuesto permite predecir la persistencia de sulfonilureas en el suelo, estimando en forma eficiente el contenido total de residuos de metsulfuron-metil y triasulfuron en el suelo a través del tiempo.

3. La degradación en el suelo de metsulfuronmetil se ajusta a una reacción cinética de primer orden, mientras que el triasulfuron se representa mejor mediante una cinética de segundo orden.

\section{REFERENCIAS}

BERGSTRÖM, L. Model predictions and field measurements of chlorsulfuron leaching under non steady state flow conditions. Pesticide Science, Chichester, v.48, p.37-45, 1996.

BLAIR, A.; MARTIN, T. A review of the activity, fate and mode action of sulfonylurea herbicides. Pesticide Science, Chichester, v.22, p.195-219, 1988.

BOESTEN, J.T.; LINDEN, A. van der. Modeling the influence of sorption and transformation on pesticide leaching and persistence. Journal of Environmental Quality, Madison, v.20, p.425-435, 1991.

CARSEL, R.; SMITH, N.; MULKEY, L.A.; DEAN, J.; JAWISE, P. User manual for the pesticide root zone model. Washington : Environmental Protection Agency, 1984. 216p.

Pesq. agropec. bras., Brasília, v.35, n.12, p.2317-2328, dez. 2000 
FUENTES, R.; CRISTI, R.; MANQUIAN, N. Persistencia de herbicidas sulfunilureas en suelos de la zona Sur de Chile: informe final proyecto FONDECYT No 1960928 .Valdivia : Universidad Austral de Chile, 1998. 50p.

HAMAKER, J. Decomposition: quantitative aspects. In: GORING, C.; HAMAKER, J.W. (Ed.). Organic chemical in the soil environment. New York : Dekker, 1972. p.253-340.

HUTSON, J.; WAGENET, R. LEACHM: leaching estimation and chemistry model, version 3.0. Ithaca : Cornell University, 1992. 150p.

JAMES, T.; KLAFFENBACH, P.; HOLLAND, P.; RAHMAN, A. Degradation of primisulfuron-methyl and metsulfuron-methyl in soil. Weed Research, Oxford, v.35, p.113-120, 1995.

KLEIN, M. The pesticide leaching model PELMO and its application. Schmallenberg : Fraunhofer Gesellschaft, 1991. 83p.

LEISTRA, M.; BROMILOW, R.; BOESTEN, J. Measured and simulated behaviour of oxamyl in fallow soils. Pesticide Science, Chichester, v.11, p.179-188, 1980.

LINACRE, E.T. A simple formula for estimating evaporation rates in various climates, using temperature data alone. Agricultural Meteorology, Amsterdam, v.18, p.409-424,1977.

NICHOLLS, P.; WALKER, A.; BAKER, R. Measurement and simulation of the movement and degradation of atrazine and metribuzin in fallow soil. Pesticide Science, Chichester, v.13, p.484-494,1982.

OLEA, F. Persistencia y movimiento de terbutilazina en tres suelos de la Región de los Lagos. Valdivia : Universidad Austral de Chile, 1996. 82p. Tesis de Licenciado en Agronomía.

SHAABAN, Z.; ELPRINCE, A.M. A simulation model for pesticide residues in a field soil. Plant and Soil, Dordrecht, v.114, p.187-195,1989.
STEEL, R.; TORRIE, J. Bioestadística: principios y procedimientos. 2.ed. México : MacGraw-Hill, 1995. $622 \mathrm{p}$.

THURN-VALSASSINA, F. Persistencia de metsulfurón-metil y triasulfurón en suelos del Sur de Chile. Valdivia : Universidad Austral de Chile, 1998. 90p. Tesis Licenciado en Agronomía.

TOSSO, J. Suelos volcánicos de Chile. Santiago : Ministerio de Agricultura, 1985. 225p.

WAGENET, R.; HUTSON, J. LEACHM: leaching estimation and chemistry model: a process based model of water and solute movement, transformation, plant uptake and chemical reaction in the unsaturated zone - continuum 2. Ithaca : Cornell University, 1987. $72 \mathrm{p}$.

WALKER, A. Evaluation of a simulation model for prediction of herbicide movement and persistence in soil. Weed Research, Oxford, v.27, p.143-152, 1987.

WALKER, A.; BARNES, A. Simulation of herbicide persistence in soil: a revised computer model. Pesticide Science, Chichester, v.12, p.123-132, 1981.

WALKER, A.; HOLLIS, J. Prediction of pesticide mobility in soil and their potential to contaminate surface and groundwater. In: HEWITT, H.G. (Ed.). Comparing glasshouse and field pesticide performance. Canterbury : University of Kent, 1994. p.211-224. (British Crop Protection Council Monograph, 59).

WALKER, A.; WELCH, S. The relative movement and persistence in soil of chlorsulfuron, metsulfuronmethyl and triasulfuron. Weed Research, Oxford, v.29, p.375-383, 1989

WALKER, A.; WELCH, S.; MELACINI, A.; MONN, H. Evaluation of three pesticide leaching models with experimental data for alachlor, atrazine and metribuzin. Weed Research, Oxford, v.36, p.37-47, 1996. 